\title{
Metabolism and Effects on Platelet Function of the Purified Eicosapentaenoic and Docosahexaenoic Acids in Humans
}

\author{
Clemens von Schacky and Peter C. Weber \\ Medizinische Klinik Innenstadt der Universität München, 8000 Munich 2, Federal Republic of Germany
}

\begin{abstract}
Metabolism and effects on platelet function of $6 \mathrm{~g} / \mathrm{d}$ for $6 \mathrm{~d}$ of either eicosapentaenoic acid (EPA, C20:5 $\omega-3)$ or docosahexaenoic acid (DCHA, C22:6 $\omega-3)$ in volunteers were compared in a randomized crossover study. Incorporation kinetics revealed that EPA appeared in plasma free fatty acids and plasma phospholipids after $4 \mathrm{~h}$, but was not incorporated into platelet phosphatidylcholine and -ethanolamine until day 6. This indicates that platelet fatty acid composition does not immediately reflect that of the surrounding plasma milieu, but rather may be determined during megakaryocyte maturation. Importantly, EPA was not incorporated into platelet phosphatidylinositol or -serine in vivo, thus reflecting selective biosynthesis of platelet phospholipids. After dietary EPA, C22:5 $\omega-3$ increased in plasma and platelet phospholipids. In contrast, DCHA-levels were unaltered. After DCHA-ingestion, $\mathrm{C20}: 5 \omega-3$ concentrations rose in plasma phospholipids, implying that retroconversion took place. These findings indicate that dietary DCHA can serve as a source of EPA. During this short-term study, ingestion of both EPA and DCHA resulted in reduced platelet aggregation in response to collagen. The response to ADP was lowered significantly only by DCHA. After either EPA or DCHA, thromboxane formation was unchanged in serum derived from clotted whole blood as was total in vivo synthesis measured by excretion of immunoreactive 2,3dinor thromboxane $B_{2 / 3}$. We conclude that DCHA reduces platelet responsiveness, contributing to the antithrombotic effects of $\omega-3$ fatty acid-rich fish oil ingestion, of which DCHA is a major component.
\end{abstract}

\section{Introduction}

Fish and fish oils, rich in the two $\omega-3$ fatty acids, eicosapentaenoic (EPA) ${ }^{1}$ and docosahexaenoic acid (DCHA), are under extensive

This study was presented in part at the 27th Annual Meeting of the American Society for Hematology, New Orleans, LA, 7-10 December 1985.

Dr. von Schacky's present address is Divisions of Hematology/Oncology, 13 West, New York VA Medical Center, 408 First Avenue, New York 10010; Dr. Weber's is Departments of Preventive Medicine and Clinical Epidemiology, Harvard Medical School, Boston, MA 02114. Received for publication 8 August 1985.

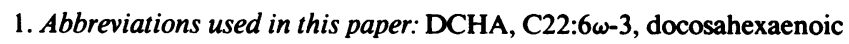
acid; EPA, C20:5 $\omega-3$, eicosapentaenoic acid; TX, thromboxane.

J. Clin. Invest.

(c) The American Society for Clinical Investigation, Inc.

0021-9738/85/12/2446/05 \$1.00

Volume 76, December 1985, 2446-2450 investigation in animals, volunteers, and patients. Their antithrombotic and antiinflammatory properties imply prophylactic and therapeutic potential (1-6). In humans, eicosanoids from EPA are less thrombogenic and less proinflammatory than those derived from arachidonic acid (7-10). Additional benefits may be attributable to the plasma lipid-lowering action of dietary fish oils and fractions derived therefrom (3,11-14). We recently carried out a detailed metabolic study of time and dose relationships of long-term dietary cod liver oil supplementation (15). These reports involved the use of fish and fish oils, i.e., mixtures of fatty acids, of which both EPA and DCHA are major constituents.

It is not known whether DCHA exerts antithrombotic effects of its own during intake of mixtures containing $\omega-3$ fatty acids. Therefore, we performed a randomized crossover study designed to compare individual metabolic and functional effects of the purified $\omega-3$ fatty acids, EPA, and DCHA in male volunteers. It will be shown that dietary DCHA can be retroconverted to EPA in humans. Interestingly, DCHA by itself reduces platelet responsiveness, and contributes therefore to the antithrombotic properties of fish and fish oils. Moreover, the results indicate that platelet phospholipid fatty acid composition may be determined during cell maturation.

\section{Methods}

Study protocol. Seven healthy male volunteers, 29-38 yr of age, weight 58-82 kg, were recruited from hospital staff. Informed consent was obtained. The volunteers were advised to take vitamin $E(100 \mathrm{mg} / \mathrm{d})$, refrain from all drug intake, and to exclude dietary fish for $1 \mathrm{wk}$ before and during the study periods. After an overnight fast, venipuncture was performed with minimal venous stasis on an antecubital vein with 19-gauge butterfly sets before and after the 6-day intake of either $6 \mathrm{~g}$ EPA or DCHA ethylester. 24-h urine samples were obtained at these time points. Four volunteers were randomized to receive EPA initially, and after a 10-wk interval the DCHA regimen was administered. One volunteer was unable to participate in his second (DCHA) phase of the study for personal reasons. In a randomized subset of four volunteers, blood was drawn for determination of fatty acid composition of plasma, phospholipids in plasma, and platelet phospholipid subclasses, before, 4,8 , and $24 \mathrm{~h}$ as well as after $6 \mathrm{~d}$ of EPA supplementation. All volunteers were unaware whether they received EPA or DCHA, and all analyses were performed without knowledge of the type of dietary supplementation.

Materials. Capsules containing $0.5 \mathrm{~g}$ of $83 \%$ pure EPA ethylester (other major component DCHA, 4.2\%), were from R/P Scherer (Eberbach, Federal Republic of Germany). Capsules containing $0.25 \mathrm{~g}$ of $90 \%$ pure DCHA ethylester (other major component C22:5w-3, 5.5\%, EPA $<1 \%$ ) were from Nippon Oil and Fats, Tokyo, Japan. The capsule content was verified by gas-liquid chromatography. All other materials have been recently described $(15,16)$.

Methods. Fatty acid analyses in plasma and of phospholipids in plasma and platelets were performed as described (15). Platelet phos- 

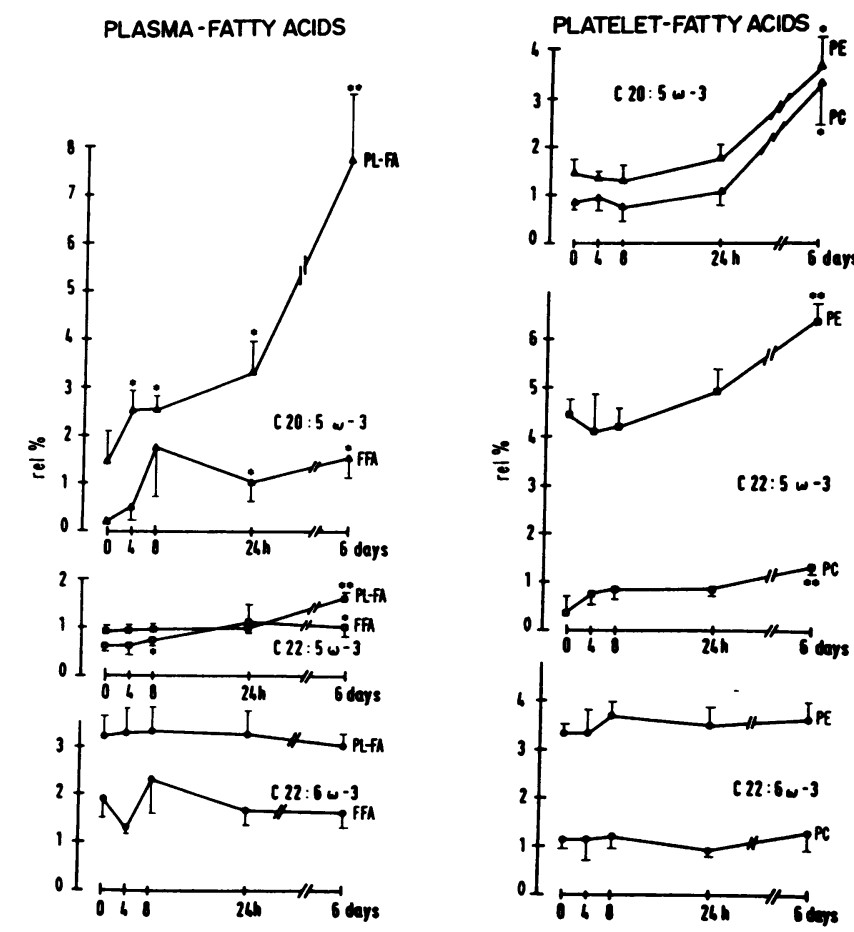

Figure 1. Incorporation of $6 \mathrm{~g}$ /day purified oral EPA into human plasma free fatty acids (FFA) and phospholipids (PL-FA) (left) and into platelet phosphatidylethanolamine (PE) and -choline (PC) (right). $n=4$; means \pm SEM. ${ }^{*} P<0.05,{ }^{* *} P<0.01$, one-tailed paired $t$ test.

phatidylethanolamine, -choline, -serine, and -inositol were separated by thin-layer chromatography (16). Fatty acid methylesters were analyzed by gas-liquid chromatography $(15,16)$. Platelet aggregation was measured in adjusted platelet-rich plasma $(250,000 / \mu \mathrm{l})$ in an aggregometer in response to $0.50 \mu \mathrm{g} / \mathrm{ml}$ collagen and $1.0 \mathrm{mM}$ ADP (15). Thromboxane (TX) $\mathrm{B}_{2 / 3}$ in whole clotted blood $\left(1 \mathrm{~h}, 37^{\circ} \mathrm{C}\right)$ and urinary 2,3 -dinor $\mathrm{TXB}_{2 / 3}$ were determined radioimmunologically (15). The paired $t$ test was applied.

\section{Results}

Fatty acid alterations. Supplementation of the diet with either EPA or DCHA for $6 \mathrm{~d}$ induced significant qualitative and quantitative changes in plasma free fatty acids and in phospholipids of plasma and platelets (Figs. 1 and 2). Despite significant increases in plasma levels of EPA, platelet EPA remained constant over a 24-h period (Fig. 1). Ingested EPA was not incorporated into platelet phosphatidylinositol or -serine at any time (detection limit of EPA was $1 \%$ relative to arachidonate). This confirms and extends our previous study with fish oil (16). In the subsequent $5 \mathrm{~d}$, levels of 20:5 $\mathrm{\omega}-3$ almost tripled in plasma phospholipids as well as in platelet phosphatidylcholine and -ethanolamine (Fig. 1).

During dietary EPA, 22:5 $\omega-3$ changed minimally in the first $24 \mathrm{~h}$, but this was followed by a significant rise in all compart-

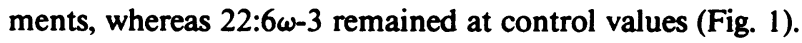

DCHA ingestion resulted in a doubling of control levels in both plasma and platelet phospholipids. However, this was accompanied by an unanticipated increase in 20:5 $\omega-3$ in plasma phospholipids, which could only have resulted from retroconversion of DCHA (Fig. 2). With regard to 22:5 -3 , a small but significant decrease in plasma and platelet phospholipids was noted during DCHA ingestion (Fig. 2).

Significant changes of any other fatty acid were only observed during EPA supplementation. Arachidonic acid decreased in plasma free fatty acids, whereas linoleic acid decreased in both plasma and platelet phospholipids (data not shown).

Platelet aggregation. As shown in Fig. 3 (left), platelet ag-
PLASMA PHOSPHOLIPID FATTY ACIDS

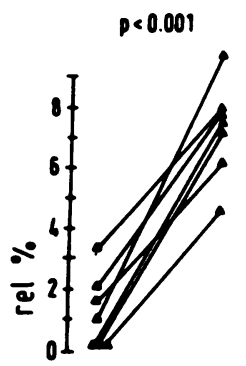

$-(20: 5 w-3$

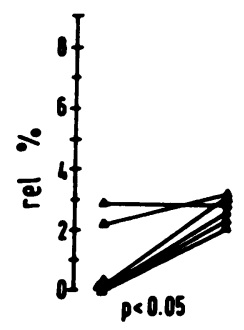

$p<0.01$

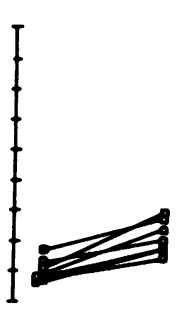

$[22: 5 w-3$

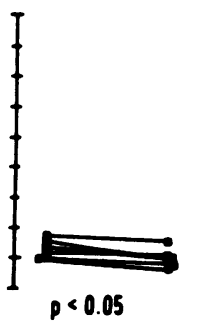

n.s.

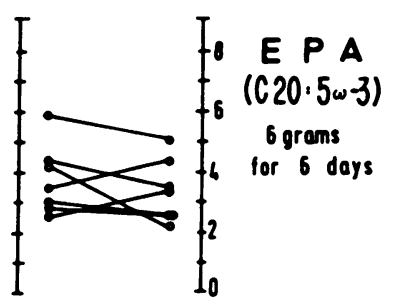

C 22:6w-3
PLATELET PHOSPHOLIPID FATTY ACIDS
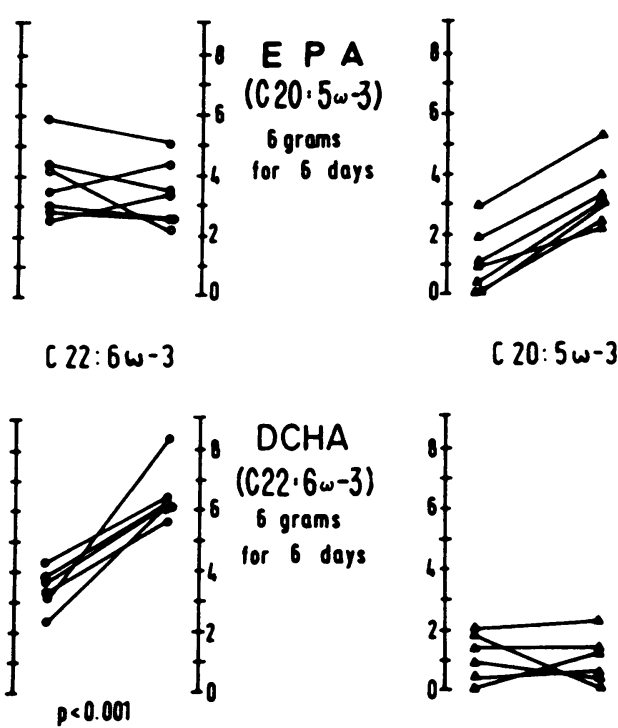

$p<0.001$

$[20: 5 \omega-3$

$p<0.05$

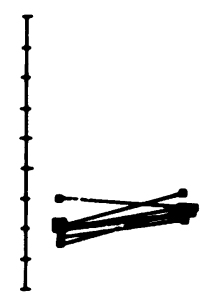

$C 22: 5 w-3$

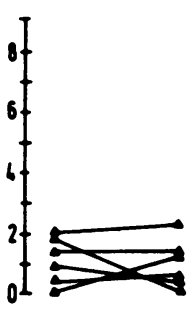

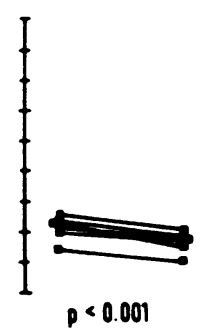

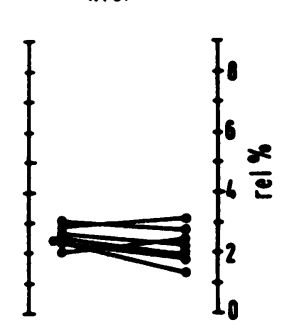

$[22: 6 \omega-3$

Figure 2. $\omega-3$ fatty acids of plasma and platelet phospholipids before and after $6 \mathrm{~g} /$ day purified oral EPA (upper panel) or DCHA (lower panel) in volunteers for $6 \mathrm{~d}$. Each volunteer is represented by one line connecting control (left) and intervention (right) value. Onetailed paired $t$ test $(P)$ was applied. 

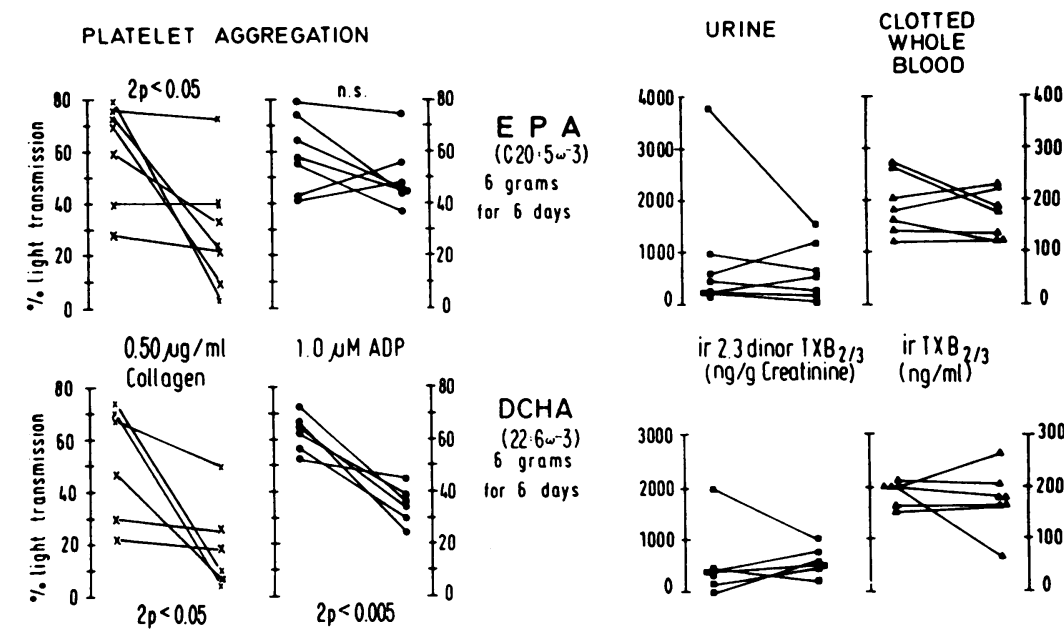

gregation ex vivo in response to collagen was reduced after both DCHA and EPA. In contrast, the response to ADP was reduced only after DCHA but not after EPA.

$T X$ formation. Formation of immunoreactive $\mathrm{TXB}_{2 / 3}$ in clotted whole blood did not change significantly after either EPA or DCHA (Fig. 3, right). The excretion rate of urinary immunoreactive 2,3-dinor $\mathrm{TXB}_{2 / 3}$ was unaltered in volunteers with normal basal excretion rates, but it decreased in the one volunteer with high control excretion rates after both EPA and DCHA.

Safety. All volunteers were repeatedly questioned concerning side effects, and no side effects were reported. Laboratory studies of blood, urine, and chemistries were unremarkable during the study.

\section{Discussion}

In this study we compared the metabolism and effects on platelet aggregation of the two major $\omega-3$ fatty acids in fish oil, EPA and DCHA individually. The major findings are: $(a)$ retroconversion of dietary DCHA to EPA took place; $(b)$ platelet responsiveness was reduced by DCHA, a major component of fish and fish oils; (c) platelet phospholipid fatty acid composition may be defined during cell maturation.

Fatty acid metabolism. The unexpected increase of 20:5 $\omega$ -

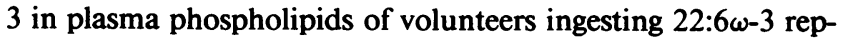
resents a retroconversion phenomenon. This has only been reported previously in the rat and in vitro $(17,18)$. Thus, DCHA may serve as a "reservoir" for EPA via partial $\beta$-oxidation (19) and saturation of the resulting trans-double bond (17).

In this study, dietary EPA was not metabolized to DCHA, which is consistent with a previous report (20). The significance of the small quantity of 22:5 $\omega-3$ observed after dietary EPA remains to be determined (21). EPA actually accumulated in plasma free fatty acids and even more so in plasma phospholipids (Fig. 1). In contrast, dietary supplementation with dihomo- $\gamma$ linolenic acid (C20:3 $\omega-6)$, found in evening primrose oil, does not lead to $20: 3 \omega-6$ accumulation, in that it is rapidly desaturated to arachidonic acid (3). Thus EPA, but not C20:3 $\omega-6$, is metabolically available over a period of time as an eicosanoid precursor after dietary intake.

$24 \mathrm{~h}$ after EPA ingestion, its content in platelet phosphati- dylethanolamine and -choline was substantially unchanged. In contrast, at this time point, EPA had already increased fivefold in plasma free fatty acids and doubled in plasma phospholipids (Fig. 1). Interestingly, $6 \mathrm{~d}$ of EPA ingestion was required before an increase in EPA content of platelet phosphatidylethanolamine and -choline was observed. These results extend our previous conclusions (15) that blood cells do not incorporate EPA from the surrounding plasma milieu in large quantities. Rather, phospholipid fatty acid composition is determined to a considerable extent during the formative stages of these cells. Alternatively, a slow rate of incorporation of fatty acids into circulating platelets, which would also be different from the much faster incorporation in vitro, could account for the above results.

EPA was not incorporated into platelet phosphatidylinositol or -serine throughout the study. This emphasizes previous observations, in which mixtures of dietary $\omega-3$ fatty acids were administered to man $(16,22,23)$. It is unlikely that short-term incorporation of EPA into phosphatidylinositol with a subsequent transacylation into phosphatidylethanolamine and -choline occurred. The selective incorporation of dietary EPA differs markedly from the in vitro situation, wherein radioactive EPA is avidly incorporated into all platelet phospholipid subclasses (cf. Reference 16), independent of the simultaneous presence of labeled arachidonic acid (24). Thus the metabolic fate of dietary EPA in vivo reflects specific control mechanisms which are undetectable in vitro.

Platelet aggregation. Dietary DCHA reduced platelet aggregation in response to both ADP and collagen. This is the first demonstration of an effect of purified dietary DCHA on platelet function in humans. Importantly, EPA levels in the platelets remained constant during DCHA intake. Therefore, reduced platelet aggregation by dietary DCHA cannot be attributed to the EPA resulting from retroconversion and, thereby, induction and formation of the inactive compound TXA $\mathrm{T}_{3}$.

In vitro, DCHA (150 $\mu \mathrm{M})$ inhibits platelet aggregation (25). Using lower concentrations $(2-10 \mu \mathrm{M})$, we did not observe such inhibition of platelet aggregation (unpublished results). Human platelets and neutrophils, enriched with dietary $\omega-3$ fatty acids, do not release detectable amounts of DCHA upon stimulation ex vivo (26), and 7- and 14-hydroxy-DCHA, the major in vitro metabolites (26-28), are only found in traces ex vivo (26). 
Therefore, interference with platelet function by hydroxy-DCHA seems unlikely. Thus, the mechanism by which DCHA reduces platelet aggregation responsiveness ex vivo remains to be clarified.

In this 6-d study, $6 \mathrm{~g}$ daily of purified dietary EPA reduced the ex vivo platelet response to collagen but not to ADP (Fig. 3). This observation was reported in another study after 2 wk of purified dietary EPA ( $3.6 \mathrm{~g} /$ day, Reference 20$)$. An additional 2 wk of EPA resulted in further inhibition of platelet responsiveness, including that to ADP (20). We conclude that dietary DCHA reduces platelet responsiveness more efficiently than EPA does. Thus, inhibition of platelet function, observed upon ingestion of dietary fish and fish oils containing both EPA and DCHA $(2-4,15,23)$, is attributable in part to DCHA.

Thromboxane formation was measured in serum from clotted whole blood, in which ex vivo platelet stimulation was presumed to be maximal. In this short-term study, neither EPA nor DCHA affected immunoreactive $\mathrm{TXB}_{2 / 3}$ formation in the test system (Fig. 3). We previously reported that, after long-term $\omega-3$ fatty acid ingestion, there was a reduction of $\mathrm{TXB}_{2}$ and immunoreactive $\mathrm{TXB}_{2 / 3}$ as measured by this test (15). Thus, duration of supplementation may be important for eicosanoid production upon maximal stimulation, in that, e.g., ionophorestimulated leukotriene $B_{4}$ production decreased after $6 \mathrm{wk}$, but was unchanged after 3-4 wk of $\omega-3$ fatty acid supplementation $(9,10)$. Consistent with our previous report utilizing long-term dietary fish oil supplementation (15), normal excretion rates of urinary 2,3 dinor- $\mathrm{TXB}_{2 / 3}$ were unaltered, whereas elevated excretion rates were reduced in this short-term study. This may be attributed to reduction of increased in vivo platelet activation by ingestion of both EPA and DCHA.

In summary, we have demonstrated, for the first time, retroconversion of dietary DCHA to EPA in humans. DCHA ingestion resulted in a greater reduction of platelet aggregation than EPA ingestion. Thus, DCHA is an important factor in the antithrombotic effects of fish oil rich in $\omega-3$ fatty acids. In addition, our results indicate that platelet fatty acid composition does not immediately reflect that of the plasma milieu, but may be defined during cell maturation.

\section{Acknowledgments}

The expert technical assistance of B. Böhlig, I. Kurzmann, and E. Schmidt is gratefully acknowledged. The authors wish to thank Dr. R. Lorenz for the computer program "Peakschema" and Dr. Aaron J. Marcus for discussing the manuscript.

This work was supported in part by the Deutsche Forschungsgemeinschaft.

\section{References}

1. Dyerberg, J., H. O. Bang, E. Stoffersen, S. Moncada, and J. R. Vane. 1978. Eicosapentaenoic acid and prevention of thrombosis and atherosclerosis. Lancet. ii:117-119.

2. Siess, W., P. Roth, B. Scherer, I. Kurzmann, B. Böhlig, and P. C. Weber. 1980. Platelet membrane fatty acids, platelet aggregation, and thromboxane formation during a mackerel diet. Lancet. i:441-444.

3. Goodnight, S. H., W. S. Harris, W. E. Connor, and D. R. Illingworth. 1982. Polyunsaturated fatty acids, hyperlipidemia, and thrombosis. Arteriosclerosis. 2:87-113.
4. Lorenz, R., U. Spengler, S. Fischer, J. Duhm, and P. C. Weber. 1983. Platelet function, thromboxane formation and blood pressure control during supplementation of the western diet with cod liver oil. Circulation. 67:504-511.

5. Kromhout, D., E. B. Bosschieter, and C. D. L. Coulander. 1985. The inverse relation between fish consumption and 20 year mortality from coronary heart disease. N. Engl. J. Med. 312:1205-1209.

6. Kremer, J. M., J. Bigauoette, A. V. Michalek, M. A. Timchalk, L. Lininger, R. I. Rynes, C. Huyck, J. Zieminski, and L. E. Bartholomew. 1985. Effects of manipulation of dietary fatty acids on clinical manifestations of rheumatoid arthritis. Lancet. i:184-187.

7. Needleman, P., A. Raz, M. D: Minkes, J. A. Ferrendelli, and H. Sprecher. 1979. Triene prostaglandins: prostacyclin and thromboxane biosynthesis and unique biological properties. Proc. Natl. Acad. Sci. USA. 76:944-948.

8. Fischer, S., and P. C. Weber. 1984. Prostaglandin $I_{3}$ is formed in vivo in man after dietary eicosapentaenoic acid. Nature (Lond.). 307: 165-168.

9. Strasser, T., S. Fischer, and P. C. Weber. 1985. Leukotriene $B_{s}$ is formed in human neutrophils after dietary supplementation with icosapentaenoic acid. Proc. Natl. Acad. Sci. USA. 82:1540-1543.

10. Lee, T. H., R. L. Hoover, J. D. Williams, R. I. Sperling, J. Ravalese, B. W. Spur, D. W. Robinson, E. J. Corey, R. A. Lewis, and K. F. Austen. 1985. Effect of dietary enrichment with eicosapentaenoic and docosahexaenoic acids on in vitro neutrophil and monocyte leukotriene generation and neutrophil function. N. Engl. J. Med. 312:1217-1224.

11. Ahrens, E. H., W. Insull, J. Hirsch, W. Stoffel, M. L. Peterson, J. W. Farquhar, T. Miller, and H. J. Thomasson. 1959. The effect on human serum-lipids of a dietary fat, highly unsaturated, but poor in essential fatty acids. Lancet. i:117-119.

12. Worne, H. E., and L. W. Smith. 1959. Effects of certain pure long chain polyunsaturated fatty acid esters on the blood lipids of man. Am. J. Med. Sci. 237:710-721.

13. Kobatabe, Y., K. Kuroda, H. Jinnouchi, E. Nishide, and S. Innami. 1984. Differential effects of dietary eicosapentaenoic and docosahexaenoic fatty acids on lowering of triglyceride and cholesterol levels in the serum of rats on hypercholesterolemic diet. J. Nuttr. Sci. Vitaminol. 30:357-372.

14. Phillipson, B. E., D. W. Rothrock, W. E. Connor, W. S. Harris, and D. R. Illingworth. 1985. Reduction of plasma lipids, lipoproteins, and apoproteins by dietary fish oils in patients with hypertriglyceridemia. N. Engl. J. Med. 312:1210-1216.

15. von Schacky, C., S. Fischer, and P. C. Weber. 1985. Long term effects of dietary marine $\omega-3$ fatty acids upon plasma and cellular lipids, platelet function and eicosanoid formation in humans. J. Clin. Invest. 76:1626-1631.

16. von Schacky, C., W. Siess, S. Fischer, and P. C. Weber. 1985. A comparative study of eicosapentaenoic acid metabolism by human platelets in vivo and in vitro. J. Lipid Res. 26:457-464.

17. Schlenk, H., D. M. Sand, and J. L. Gellerman. 1969. Retroconversion of docosahexaenoic acid in the rat. Biochim. Biophys. Acta. 187: 201-207.

18. Yorek, M. A., R. R. Bohnker, D. T. Dudley, and A. A. Spector. 1984. Comparative utilization of $n-3$ polyunsaturated fatty acids by cultured human Y-79 retinoblastoma cells. Biochim. Biophys. Acta. 795: 277-285.

19. Stoffel, W., W. Ecker, H. Assad, and H. Sprecher. 1970. Enzymatic studies on the mechanism of the retroconversion of $\mathrm{C} 22$-polyenoic fatty acids to their C20 homologues. Z. Physiol. Chem. 351:1545-1554.

20. Terano, T., A. Hirai, T. Hamazaki, S. Kobayashi, T. Fujita, Y. Tamura, and A. Kumagai. 1983. Effect of oral administration of highly purified eicosapentaenoic acid on platelet function, blood viscosity, and red cell deformability in healthy human subjects. Atherosclerosis. 46: 321-331.

21. Careaga, M. M., and H. Sprecher. 1984. Synthesis of two hydroxy 
fatty acids from 7,10,13,16,19-docosapentaenoic acid by human platelets. J. Biol. Chem. 259:14413-14417.

22. Ahmed, A. A., and B. J. Holub. 1984. Alteration and recovery of bleeding times, platelet aggregation and fatty acid composition of individual phospholipids in platelets of human subjects receiving a supplement of cod liver oil. Lipids. 19:617-624.

23. Galloway, J. H., I. J. Cartwright, B. E. Woodcock, M. Greaves, R. G. G. Russel, and F. E. Preston. 1985. Effect of dietary fish oil supplementation on the fatty acid composition of the human platelet membrane: demonstration of selectivity in the incorporation of eicosapentaenoic acid into membrane phospholipid pools. Clin. Sci. 68:449-454.

24. Weiner, T. W., and H. Sprecher. 1985. 22-carbon polyenoic acids. Incorporation into platelet phospholipids and the synthesis of these acids from 20-carbon polyenoic acid precursors by intact platelets. J. Biol. Chem. 260:6032-6038.
25. Rao, G. H. R., E. Radha, and J. G. White. 1983. Effect of docosahexaenoic acid (DHA) on arachidonic acid metabolism and platelet function. Biochem. Biophys. Res. Commun. 117:549-555.

26. Fischer, S., C. von Schacky, W. Siess, T. Strasser, and P. C. Weber. 1984. Uptake, release and metabolism of docosahexaenoic acid (DHA, C22:6w-3) in human platelets and neutrophils. Biochem. Biophys. Res. Commun. 120:907-918.

27. Aveldano, M. I., and H. Sprecher. 1983. Synthesis of hydroxy fatty acids from $4,7,10,13,16,19\left(1-{ }^{14} \mathrm{C}\right)$ docosahexaenoic acid by human platelets. J. Biol. Chem. 258:9339-9343.

28. Lee, T. H., J. M. Mencia-Huerta, C. Shih, E. J. Corey, R. A. Lewis, and K. F. Austen. 1984. Effects of exogenous arachidonic, eicosapentaenoic, and docosahexaenoic acids on the generation of 5-lipoxygenase pathway products by ionophore-activated human neutrophils. J. Clin. Invest. 74:1922-1933. 